\title{
The Application of Laser-Assisted Indocyanine Green Fluorescent Dye Angiography in Microsurgical Breast Reconstruction
}

\author{
Martin I. Newman, M.D., F.A.C.S., ${ }^{1}$ and \\ Michel C. Samson, M.D., F.R.C.S.(C), F.A.C.S. ${ }^{1}$
}

The benefits of laser-assisted indocyanine green fluorescence angiography have previously been demonstrated in cardiac surgery. The purpose of this study was to determine the value of this technology in microsurgical breast reconstruction. Intraoperative laser-assisted indocyanine green fluorescence angiography was performed on all microsurgical breast reconstruction cases (deep inferior epigastric perforator flap or free transverse rectus abdominus muscle flap) during the study period. Ten consecutive free tissue transfer autologous breast reconstructions were performed on 8 women. In four cases, imaging demonstrated flow or perfusion deemed "marginal" or "poor" by the operating surgeons. In three of these cases, one involving poor arterial inflow, one of poor venous outflow, and one of poor perfusion of a mastectomy flap, the intraoperative plan was adjusted accordingly and follow-up imaging demonstrated improvement. In the fourth case, no adjustment was made at operation. However this patient required a return to the operating room for venous congestion of the flap, which was corrected without sequela. Overall flap survival was $100 \%$. We concluded that laser-assisted indocyanine green fluorescence angiography appears to provide important information that has helped guide intraoperative decision making in our series.

KEYWORDS: Breast, microsurgery, reconstruction, angiography, indocyanine green fluorescence

Free tissue transfer, primarily using the deep inferior epigastric perforator (DIEP) flap or the free transverse rectus abdominus muscle (TRAM) flap, represents the state of the art for breast reconstruction. ${ }^{1}$ Although this method does yield excellent aesthetic results ${ }^{2}$ and clinical outcomes, ${ }^{3}$ the technical complexity of the procedure entails that surgical complications, although relatively low, are not uncommon. Nahabedian et $\mathrm{al}^{4}$ reported the outcomes on 163 free TRAM or DIEP flap reconstructions performed on 135 women. Of the 143 free TRAM flaps, complications included return to the operating room (7.7\%), total necrosis (3.5\%), mild fat necrosis (9.8\%), and mild venous congestion (1.4\%). Complications in the 20 DIEP flaps included return to
${ }^{1}$ Department of Plastic and Reconstructive Surgery, Cleveland Clinic Florida, Weston, Florida.

Address for correspondence and reprint requests: Martin I. Newman, M.D., F.A.C.S., Department of Plastic and Reconstructive Surgery, Cleveland Clinic Florida, 2950 Cleveland Clinic Blvd., Weston, FL 33331 (e-mail: newmanm@ccf.org).
J Reconstr Microsurg 2009;25:21-26. Copyright (C) 2009 by Thieme Medical Publishers, Inc., 333 Seventh Avenue, New York, NY 10001, USA. Tel: +1(212) 584-4662.

Received: March 26, 2008. Accepted: April 2, 2008. Published online: October 15, 2008.

DOI 10.1055/s-0028-1090617. ISSN 0743-684X. 
the operating room (15\%), total necrosis (5\%), and mild fat necrosis (10\%). The same group also reported a more detailed analysis of the factors contributing to anastomotic failure. ${ }^{5}$ In this series of 240 flaps, $20(8.3 \%)$ required return to the operating room, with total necrosis occurring in $9(3.8 \%)$ and salvage possible in the remaining 11 flaps. Venous occlusion was the responsible factor for 16 out of the 20 returns and 8 of the 9 flap failures. Similarly, Gill et al reported on outcomes of a very large series of DIEP flaps. ${ }^{6}$ They retrospectively analyzed outcomes on 758 flaps performed over a 10-year period. Partial flap loss occurred in $19(2.5 \%)$ cases with total flap loss in $4(0.5 \%)$. Patients with 29 flaps (3.8\%) were returned to the operating room because of venous congestion. The anastomotic problems are generally recognized after the patient leaves the operating room, requiring a return to the operating room with occasional loss of the flap. It is likely that the occurrence of these complications could be reduced if potential problems were identified and corrected before the patient leaves the operating room.

We became aware of an intraoperative imaging technique that is being used routinely to assess the integrity of bypass graft anastomoses and related perfusion by the cardiac surgeons at our institution and sought to determine if this technology could provide similar clinical utility during breast reconstruction. Laser-assisted indocyanine green (LAICG) angiography uses the fluorescence properties of ICG to acquire real-time images of the coronary vasculature and bypass grafts prior to closing the chest and thus permitting revision of suspect anastomoses before the patient leaves the operating room. The clinical usefulness of this imaging technology in cardiac surgery has been the subject of several reports in the literature. ${ }^{7,8}$

A recent report has also suggested a role for this technology in the plastic and reconstructive arena. In 2002, LA-ICG fluorescence was examined as a new method for evaluating skin perfusion and found to be superior to conventional fluorescein angiography. ${ }^{9}$ In this prospective clinical study, LA-ICG fluorescence videoangiography was used for the intraoperative evaluation of skin-flap perfusion. The results of LA-ICG imaging were compared with clinical outcome 1 week postoperatively. In that study, the authors found that intraoperative LA-ICG filling defects were associated with delayed wound healing in each case.

Based on the preceding, we set out to evaluate the role of laser-assisted intraoperative ICG angiography in free tissue transfer reconstructive surgery as a means to evaluate the microcirculation of donor and recipient vessels as well as overall tissue perfusion of the free flaps designed for transfer.

\section{METHODS}

\section{Patient Selection and Surgery}

Institutional review board approval was sought and granted for use of the SPY imaging system (Novadaq Technologies Inc., Toronto, Canada) during free tissue breast reconstructive surgery. All study participants signed informed consent before surgery. Ten ${ }^{10}$ consecutive free tissue transfer autologous breast reconstruction cases on eight female patients were included in the study. The average age was 52.3 years. Recipient vessels included nine thoracodorsal pedicles and one internal mammary pedicle in a patient who had previously undergone axillary dissection and radiation therapy.

\section{Details of Imaging}

Intraoperative images were acquired using the SPY imaging system. The Food and Drug Administration has cleared this system for use in coronary artery bypass graft surgery and, subsequent to the initiation of this study, has also cleared it for plastic and reconstructive surgery. The system employs the fluorescence properties of ICG (IC-Green; Akorn Inc., Buffalo Grove, IL). When illuminated at $806 \mathrm{~nm}$, ICG fluoresces, emitting light of longer wavelengths centered at $\sim 830 \mathrm{~nm}$. Following intravenous injection, ICG is rapidly and extensively bound to plasma proteins and so is retained within the vasculature, a property that makes it an ideal agent for acquiring images of blood vessels in vivo. The imaging system consists of an 806-nm laser light source and a camera equipped with appropriate optical filters. These components are housed within an imaging head connected to a mobile cart (housing the computer, etc.) by means of an articulated arm. The imaging head, enclosed in a sterile drape, is positioned 12 inches above the area of interest. The ICG is administered as a bolus through a venous line (central or peripheral), and images are acquired during the first pass of the bolus through the field of view. The images, acquired at a rate of 30 per second, are displayed in real time on a monitor permitting immediate evaluation in the operating room and are saved to computer disk for future review and archiving.

In each case, intraoperative angiography was performed at several strategic points during the case. After dissection of the flaps and the pedicles, but before actual flap harvest, ICG angiography was performed on the donor pedicles and on the skin and subcutaneous tissue comprising the flap. Following free tissue transfer and microsurgical anastomosis of both the artery and vein(s), LA-ICG angiography was again performed visualizing the postanastomotic circulation through the artery and vein(s) as well as the skin and subcutaneous tissue of the flap. Finally, ICG imaging of the flaps, from the skin side, following inset was performed for each flap. Additional visualization of the mastectomy flaps was also obtained. 
The concentration of ICG injected was constant throughout the study at $2.5 \mathrm{mg} / \mathrm{mL}$. However, the volume of ICG injected varied depending on the site selected for imaging. For imaging of exposed vessels such as the pre- or postanastomotic pedicles, a total of $2 \mathrm{~mL}(5 \mathrm{mg})$ was injected intravenously. For imaging of skin and subcutaneous tissue, $4 \mathrm{~mL}$ (10 mg) of ICG was injected.

\section{RESULTS}

The eight patients enrolled in the study were all imaged successfully. Flap survival was $100 \%$. One patient who underwent bilateral breast reconstruction with bilateral DIEP flaps was returned to the operating room in the first 24 hours for venous congestion in one of the flaps (see later discussion).

A total of 87 intraoperative images were acquired. These included 50 angiograms (5 angiograms for each of 10 flaps) as follows ${ }^{1}$ : arterial and venous pedicle prior to harvest ${ }^{2}$; subcutaneous underside of flap prior to harvest $^{3}$; skin side of flap prior to harvest ${ }^{4}$; arterial and venous pedicle following anastomosis ${ }^{5}$; and skin side of flap following inset. Figures 1 to 5 show examples of these images. These figures show static images extracted from a movie (or cine) sequence and thus provide a snapshot in time from the entire sequence. Viewing the original movie sequence provides the additional benefit of being able to (subjectively) assess the dynamic nature of the flow and perfusion in each case.

In Fig. 1, both the arterial and venous flow can be seen in the pedicle prior to harvest. This static image was from a relatively late point in the image sequence when the arterial inflow was starting to fade and the venous outflow was just becoming evident. Figures 2 and 3 demonstrate full perfusion of the subcutaneous under-

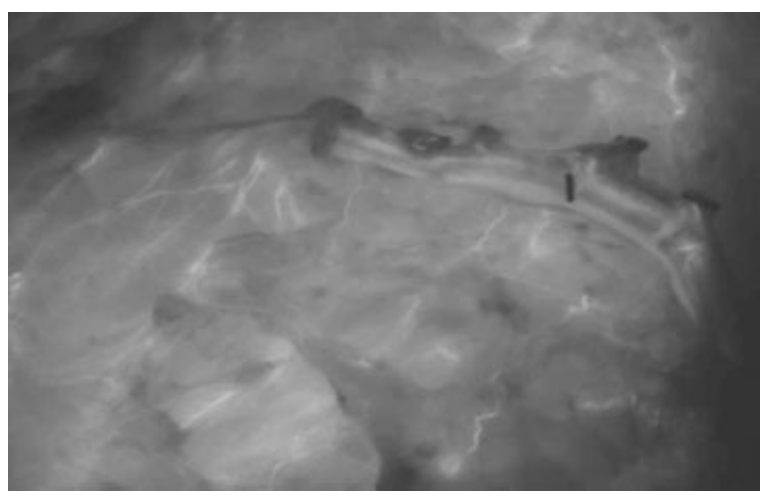

Figure 1 Image of the arterial inflow and venous outflow in a deep inferior epigastric perforator flap prior to harvest. The image sequence was acquired following injection of $5 \mathrm{mg}$ indocyanine green into the central venous line. This static image is from late in the sequence and shows the late phase of arterial filling (upper blood vessel) coincident with the early phase of venous outflow (lower vessel). The dark bar in the image is due to the presence of a vascular clip.

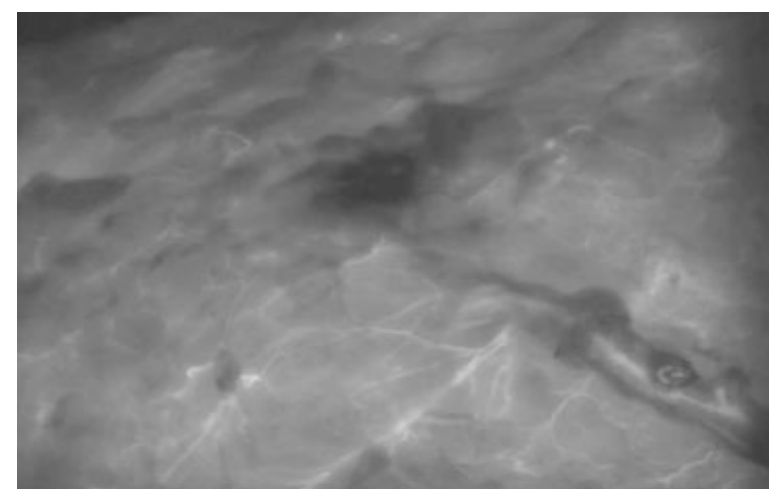

Figure 2 Image of the subcutaneous aspect of a deep inferior epigastric perforator flap prior to harvest. The image sequence was acquired following injection of $10 \mathrm{mg}$ indocyanine green into the central venous line and demonstrates good perfusion over the entire body of the flap.

side and the skin side of the flap, respectively, prior to harvest. In both cases excellent perfusion is observed over the entire surface of the flap including the outermost borders. Figure 4 is an example of an image acquired following completion of the anastomoses and demonstrates good arterial inflow and venous return through the pedicles. Figure 5 is an image acquired following completion of the flap and demonstrates adequate perfusion out to the full border of the flap.

An additional 33 images were acquired as repeats of these standard image sequences. Repeat images were acquired in situations where there was difficulty in visualizing flow in both the arterial and venous phases of the same pedicle (the system is currently limited to 34 seconds of image acquisition, which is often not long enough to capture both phases) or there was concern about the adequacy of flow or perfusion. Five ${ }^{5}$ additional images were captured in an effort to evaluate the perfusion of the mastectomy flaps, which displayed a marginal clinical appearance.

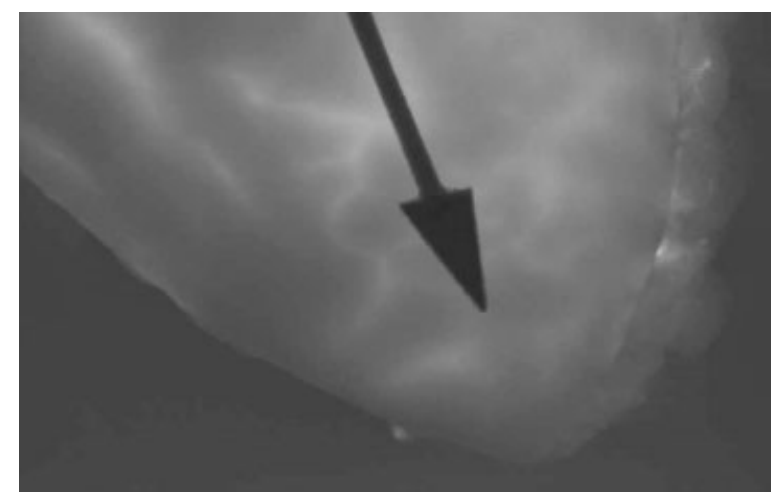

Figure 3 Image of the skin side of a deep inferior epigastric perforator flap prior to harvest. The image sequence was acquired following injection of $10 \mathrm{mg}$ indocyanine green into the central venous line and demonstrates good perfusion over the entire flap. Arrow points to tip of flap, indicating adequate perfusion. 


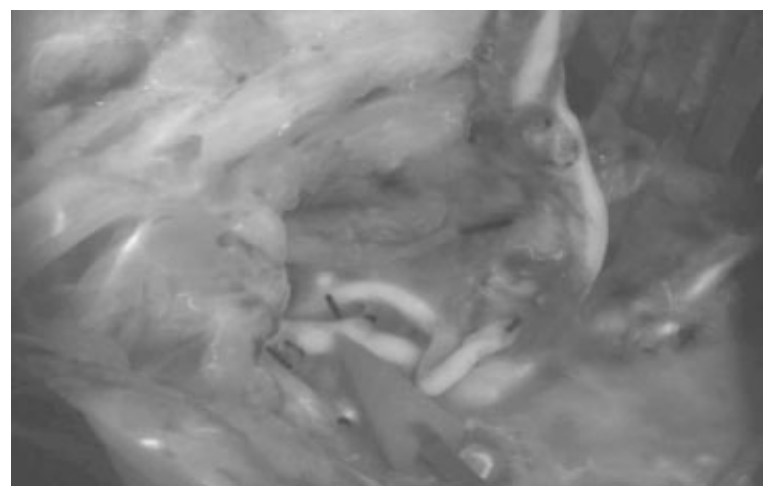

Figure 4 Image of the arterial and venous pedicles upon completion of the anastomoses. The image sequence was acquired following injection of $5 \mathrm{mg}$ indocyanine green into the central venous line. This static image is from late in the image sequence and demonstrates the late phase of arterial inflow coincident with excellent venous outflow.

Of the 87 image sequences acquired, 78 (89.7\%) demonstrated adequate flow and perfusion as determined subjectively by the operating surgeons at the time of capture. Nine (10.3\%) images demonstrated flow or perfusion that was subjectively considered as inadequate or poor as determined by the operating surgeons at the time of capture. Of the eight patients studied, images from four were in this latter category as a cause of concern to the surgeon. These included three repeat images of marginal venous return during harvest of one flap, one repeat image of poor arterial inflow prior to harvest, two images of delayed venous return following right internal mammary vein anastomosis, and three images of one particular mastectomy flap.

In three of the cases, images considered subjectively to demonstrate inadequate flow or poor perfusion as determined by the operating surgeons at the time of

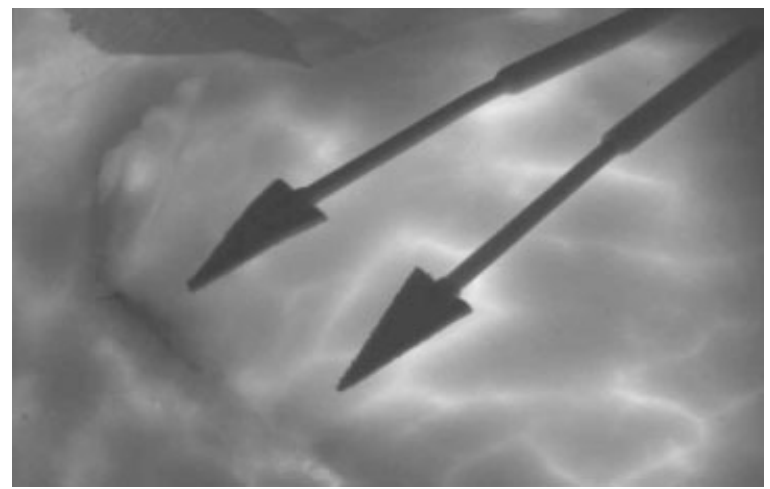

Figure 5 Image of the skin side of a deep inferior epigastric perforator flap upon completion. The image sequence was acquired following injection of $10 \mathrm{mg}$ indocyanine green into the central venous line. The arrows overlay the flap and point to the suture line. This image demonstrates excellent perfusion over the entire flap, extending up to the suture line, and also equal perfusion of the skin in the donor site. capture stimulated the surgeons to alter the operative plan. In one case the surgeons elected not to change the operative plan. Ultimately, this proved to be the flap that demonstrated significant venous congestion within the 24 hours following surgery, requiring a return to the operating room.

\section{CASE 1}

\section{Marginal Venous Return Prior to Harvest}

In this case the initial image of the arterial and venous pedicles prior to harvest demonstrated adequate arterial inflow but marginal venous return (Fig. 6). Repeat images acquired following repositioning of the flap, to ensure there was no kinking or other physical obstruction of the vessel, confirmed this assessment. An additional perforator was identified and included in the flap. Reimaging of the flap confirmed improved venous return. The case was completed with further imaging, confirming adequate arterial inflow, venous return, and subcutaneous perfusion of the completed flap.

\section{CASE 2}

\section{Inadequate Arterial Inflow Prior to Harvest}

The second case in which imaging revealed a potential cause for concern was one in which the preharvest image demonstrated inadequate arterial inflow (Fig. 7). The area between the arrows in the panel on the left remained dark throughout the image sequence, suggesting poor arterial

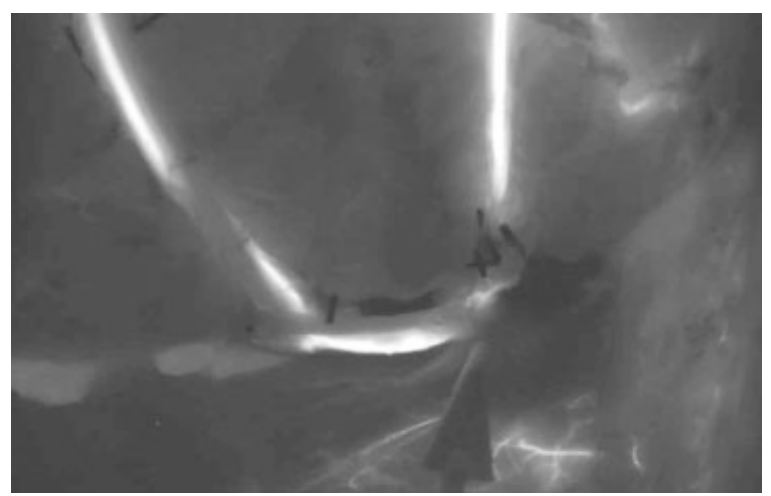

Figure 6 Example of a deep inferior epigastric perforator flap exhibiting poor venous outflow. The image sequence was acquired prior to harvesting the flap. The brightly fluorescing artery is partly overlaid by the dark vein, which contains no indocyanine green. Continuation of image acquisition did show some very sluggish venous outflow but only after the arterial fluorescence had greatly diminished (see Fig. 1, which demonstrates excellent arterial inflow and venous outflow, in which case fluorescence can be observed simultaneously in both vessels). The problem was corrected by including an additional perforator in the flap prior to harvest. 

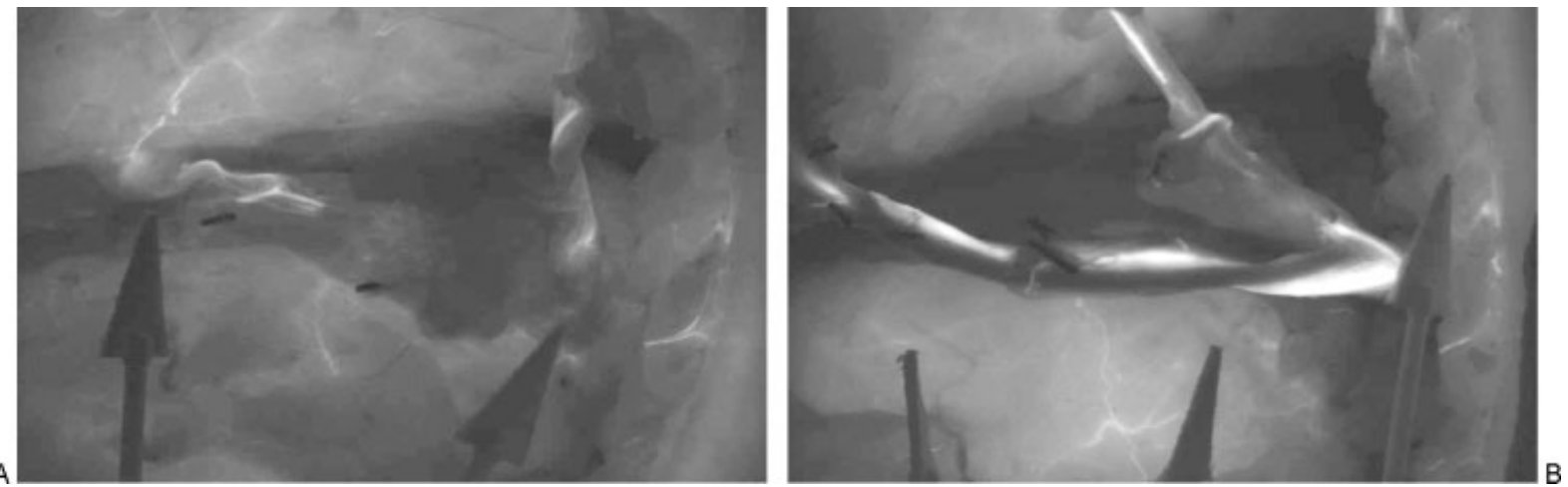

Figure 7 Example of a deep inferior epigastric perforator flap exhibiting poor arterial inflow prior to harvest. (A) The dark area between the arrows indicates poor arterial inflow. A second perforator was then included in the flap. (B) This image is from early in the arterial filling phase and demonstrates excellent arterial filling. The venous outflow has not yet started, and so the veins remain dark.

inflow. As with the case involving marginal venous return in a flap, the flap was reimaged following repositioning to rule out kinking or other physical obstruction of the artery. The second image confirmed the finding of inadequate arterial inflow; further dissection was performed and an additional perforator was identified and included in the flap. Adequate arterial inflow following inclusion of this additional perforator is demonstrated in the panel on the right. The case was completed uneventfully with further imaging confirming good flap function, and the postoperative course was uneventful.

\section{CASE 3}

\section{Poor Perfusion of a Mastectomy Flap}

In this case, on completion of the mastectomy, imaging of the mastectomy flap revealed poor perfusion at the

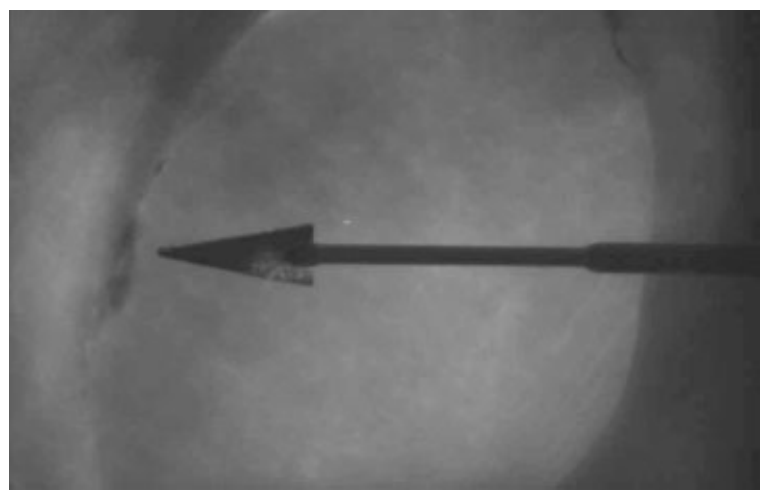

Figure 8 Example of poor perfusion in a mastectomy flap. Imaging of the deep inferior epigastric perforator (DIEP) flap following completion of the arterial and venous anastomoses revealed excellent perfusion of the flap. However, very little perfusion was observed in the border of the mastectomy flap as seen by the lack of fluorescence in the area at the extreme right of the image. The nonperfused area of the mastectomy flap was debrided before complete insertion of the DIEP flap. The arrow overlies the DIEP flap. Its tip points to the junction between the DIEP flap and the mastectomy flap. border of this flap (Fig. 8). The area of questionable perfusion was debrided, subsequent imaging demonstrated adequate perfusion to the full limits of the mastectomy flap, and the case was completed without further issues of concern.

\section{CASE 4}

\section{Delayed Venous Return in Bilateral Deep Inferior Epigastric Perforator}

The fourth case of interest was a patient who underwent bilateral breast reconstruction with bilateral DIEP flaps. When postanastomosis venous return was noted to be delayed following right internal mammy vein anastomosis, the surgeons elected not to change the operative plan. This flap demonstrated significant venous congestion within the 24 hours following surgery, requiring a return to the operating room. At reoperation, an extrinsic compression was noted on the proximal right internal mammary vein at the site of an adjacent costal cartilage, which was trimmed to relieve the obstruction and restore the flow. The anastomosis did not require revision.

\section{DISCUSSION}

Both surgeons and patients alike appreciate the benefits of autologous tissue reconstruction over prosthetic reconstruction. ${ }^{1,2}$ However, free-tissue transfer with microsurgical anastomosis is technically demanding and time consuming. In the past, microsurgeons have adopted a variety of tools and techniques designed to reduce operative time as well as a host of postoperative monitoring devices to confirm the viability of transferred tissue in the immediate postoperative phase.

Here we have performed a feasibility study on the use of LA-ICG angiography for on-table assessment of flap perfusion. ICG has a long and safe history of use in 
human medicine starting in the middle of the last century. The optical properties of ICG made it a very useful agent for dye dilution studies for measuring cardiac output. Similarly its excretion exclusively by the liver led to its widespread use for assessing hepatic function. The fluorescence properties of ICG were recognized by Flower ${ }^{10}$ in the 1970s and led to its use for angiography of the choroidal circulation in the eye. More recently its fluorescence properties have been exploited to provide intraoperative images during coronary artery bypass graft surgery. ${ }^{7,8}$ Throughout the years of experience with ICG the incidence of adverse effects of the dye have been relatively infrequent, and they have generally been of low severity. ${ }^{11,12}$

In this case series, we have gained an initial experience with the application of LA-IGF angiography in free tissue transfer breast reconstruction surgery. Overall, surgeons greatly appreciated the ability to visualize arterial inflow and venous return prior to harvest and following anastomosis. In contrast to more subjective tests performed intraoperatively, such as the venous fill test, objective visual evidence of perfusion in real time as demonstrated by the angiograms clearly confirmed arterial inflow, venous return, and skin perfusion.

One of the more significant finding in our series regarding the usefulness of this modality was the fact that when delayed venous flow following anastomosis to the right internal mammary vein was identified, failure to correct this problem intraoperatively resulted in venous congestion in the immediate postoperative phase, requiring return to the operating room as discussed earlier.

With attention to the preceding, we believe that LA-IGF angiography adds significantly to the multitude of clinical factors at the disposal of the microsurgeon. As such, we have adopted it into our practice and perform this imaging routinely during all our microsurgical cases. In an effort to evaluate the usefulness of this modality on clinical outcome in a statistically significant manner, we recommend a prospective multicenter trial.
NOTE

Both Drs. Newman and Samson serve as consultants to Novadaq Technologies, the manufacturer of the device used in this study.

\section{REFERENCES}

1. Serletti JM, Moran SL. Microvascular reconstruction of the breast. Semin Surg Oncol 2000;19:264-271

2. Tonseth KA, Hokland BM, Tindholdt TT, Abyholm FE, Stavem K. Patient-reported outcomes after breast reconstruction with deep inferior epigastric perforator flaps. Scand J Plast Reconstr Surg Hand Surg 2007;41:173-177

3. Lipa JE. Breast reconstruction with free flaps from the abdominal donor site: TRAM, DIEAP and SIEA flaps. Clin Plast Surg 2007;34:105-121

4. Nahabedian MY, Momen B, Galdino G, Manson PN. Breast reconstruction with free TRAM or DIEP flap: patient selection, choice of flap and outcome. Plast Reconstr Surg 2002;110:466-475

5. Nahabedian MY, Momen B, Manson PN. Factors associated with anastomotic failure after microvascular reconstruction of the breast. Plast Reconstr Surg 2004;114:74-82

6. Gill PS, Hunt JP, Guerra AB, et al. A 10-year retrospective review of 758 DIEP flaps for breast reconstruction. Plast Reconstr Surg 2004;113:1153-1160

7. Taggart DP, Choudhary B, Anastasiadis K, Abu-Omar Y, Balacumaraswami L, Pigott DW. Preliminary experience with a novel intraoperative fluorescence imaging technique to evaluate the patency of bypass grafts in total arterial revascularization. Ann Thorac Surg 2003;75:870-873

8. Desai ND, Miwa S, Kodama D, et al. Improving the quality of coronary bypass surgery with intraoperative angiography: validation of a new technique. J Am Coll Cardiol 2005;46: $1521-1525$

9. Holm C, Mayr M, Haofter E, Becker A, Pfeiffer UJ, Muhlbauer W. Intraoperative evaluation of skin-flap viability using laser-induced fluorescence of indocyanine green. Br J Plast Surg 2002;55(55):635-644

10. Flower RW, Hochheimer BF. A clinical technique and apparatus for simultaneous angiography of the separate retinal and choroidal circulations. Invest Ophthalmol 1973;12:248-261

11. Benya R, Quintana J, Brundage B. Adverse reactions to indocyanine green: a case report and a review of the literature. Cathet Cardiovasc Diagn 1989;17:231-233

12. Hope-Ross M, Yannuzzi LA, Gragoudas ES, et al. Adverse reactions due to indocyanine green. Ophthalmology 1994; 101:529-533 